\section{Acute presentation of vascular disease within the orbit-a descriptive synopsis of mechanisms}

\begin{abstract}
Vascular events in the orbit can present with an acute onset of symptoms, and, if untreated, raised orbital pressure and reduced arterial perfusion can lead to loss of orbital functions. Such events are commonly due to haemorrhage, but can also be due to arterio-venous shunts and very rarely arise from intraorbital vascular occlusion. The likely diagnosis is often evident on taking a thorough history and examination and in most cases, after appropriate imaging, the process can be monitored for progression. Visual failure is usually due to high pressure at the orbital apex, resulting in ischaemic optic neuropathy, and any evidence of a persistent significant visual impairment should prompt intervention. Eye (2013) 27, 299-307; doi:10.1038/eye.2012.283; published online 1 February 2013
\end{abstract}

Keywords: Orbit; vascular; arterio-venous anomaly; haemorrhage; lymphangioma; haemangioma; varix

\section{Introduction}

Vascular events in the orbit can present with an acute onset of symptoms: in some cases this presentation leads to a new diagnosis of underlying vascular disease, whereas in other cases it can be an acute deterioration of the signs or symptoms where underlying vascular disease was already known. A sudden onset of vascular-related symptoms and signs is generally due to orbital haemorrhage, acute arterio-venous shunting, or subacute thrombosis (typically within orbital veins) with resultant local changes in orbital perfusion.

Typical symptoms and signs of an acute vascular event within the orbit include a sudden onset of proptosis-commonly overnight-but, in many cases, periocular bruising might not

GE Rose and DH Verity

appear for a few days after onset of symptoms (Figure 1a). Mediated through the oculo-cardiac vagal reflex, a sudden stretching of the extraocular muscles may lead to severe pain, nausea or vomiting - this being particularly troublesome in children; in such cases, the haemorrhage can be incorrectly attributed to the vomiting (rather than vice-versa). The acute onset of extravascular haemorrhage or intravascular pathology, particularly when at the orbital apex, often leads to an embarrassment of the posteriorly-directed venous drainage; this manifests as episcleral venous congestion (Figure 1b) and a secondary rise in intraocular pressure that, in cases of significant arterio-venous shunting, will often show an abnormally high variation during the cardiac cycle (as high as $10 \mathrm{mmHg}$ ). Fluid leakage from abnormal orbital vessels also increases the intercellular fluid, this presenting clinically as a generalised impairment of eye movements (due to oedematous muscles), as caruncular or subconjunctival oedema

(Figure 1c) and, very rarely, with frank bleeding from the orbit (Figure 1d). Rarely, patients are aware of a sudden onset of orbital pulsation or a 'rushing sound' in their head.

\section{Acute orbital haemorrhage}

Spontaneous orbital haemorrhage from normal orbital vessels is extremely rare, but can occur during extreme Valsalva manoeuvre-such as might occur during delivery or with extreme exertional sports, such as power-lifting-or after extreme venous congestion due to, for example, severe vomiting or strangulation (Table 1). ${ }^{1-5}$ Haemorrhages from normal vasculature generally arises when small vessels perforating the orbital walls are disrupted by fractures and, in some cases, the site of blow can be outside the orbit (Figures 2a and b).
Orbital Clinic, Moorfields Eye Hospital, London, UK

Correspondence: GE Rose, Orbital Clinic, Moorfields Eye Hospital, City Road, London EC1V 2PD, UK. Tel: $+44(0) 2072533411$; Fax: +44 (0)20 7566 2608; E-mail: geoff.rose@ moorfields.nhs.uk

Received: 22 March 2012 Accepted: 23 March 2012 Published online: 1 February 2013 

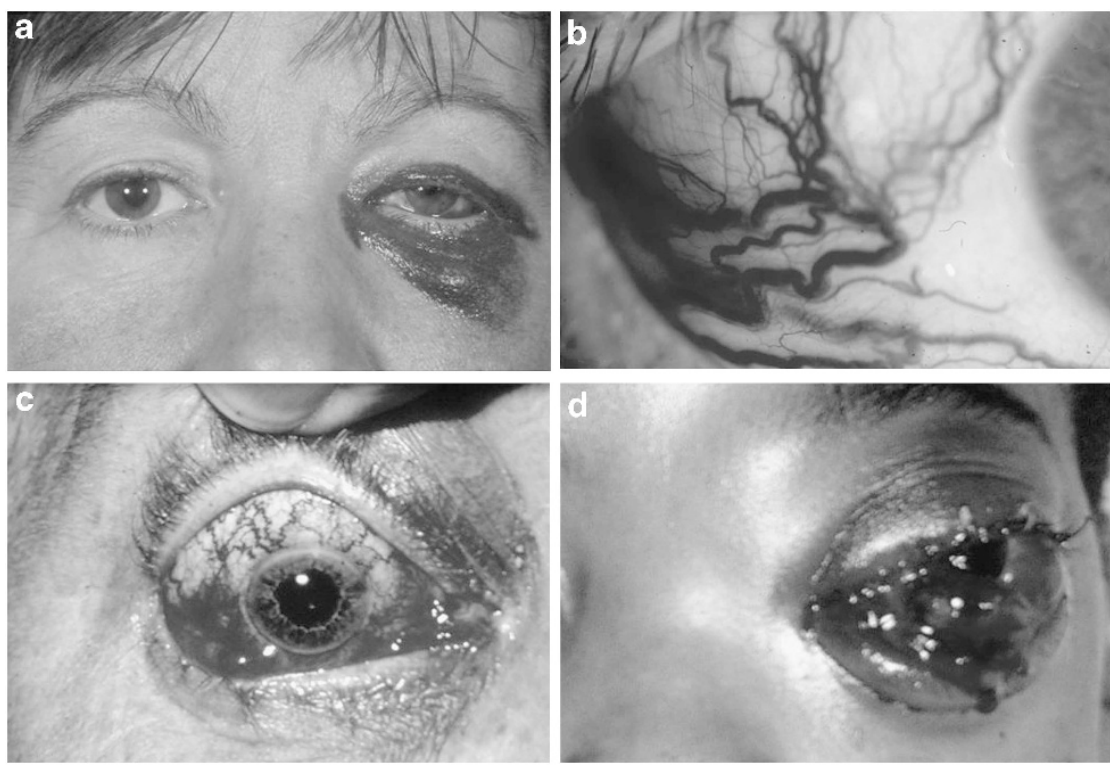

Figure 1 (a) Patient in whom periocular bruising appeared 3 days after overnight onset of left orbital pain and minor proptosis. (b) Chronic right episcleral venous congestion due to an orbital mass encroaching on the superior orbital fissure, (c) Leakage of fluid into the right orbit due to raised intravascular pressure, secondary to a low-flow dural shunt. (d) Massive orbital haemorrhage and conjunctival bleeding due to acute high-pressure vascular shunting in the left orbit.

Table 1 Predisposing factors and sources for acute orbital haemorrhage

From normal vessels

Spontaneous haemorrhage from normal vessels is very rare

During extreme Valsalva manoeuvre (for example, delivery, vomiting or weight-lifting)

Most acute haemorrhages are during orbital or periorbital trauma

From diseased, but previously normal, blood vessels

Quite common in patients with hypertension and/or diabetes Very frequently the patient is on anti-thrombotic drugs (anti-platelet agents)

From congenital or acquired periorbital vascular malformations Common in venous-lymphatic malformations

Rare in arterio-venous malformations

Almost never with acquired low-flow 'dural' arterio-venous shunts

Rare with acquired high-flow carotico-cavernous fistulae

From neoplastic vessels

Haemorrhage in (from) tumours is distinctly rare

The commonest cause of orbital haemorrhage is spontaneous rupture of the fragile microvasculature of the elderly, especially those on aspirin or other antiplatelet agents, and in younger patients with chronic diabetes or longstanding (or uncontrolled) hypertension. Presentation in such cases tends to be with a rapid onset of unilateral proptosis-often on waking-and minimal disruption of vision or eye movements (Figure 2c); occasionally the patient will remember a precipitating event, such as a severe bout of coughing, just prior to onset of symptoms. On imaging, these spontaneous arteriopathic haemorrhages typically lie in the posterior half of the infero-temporal quadrant and assume a 'beached whale' configuration along the lateral wall (Figure 2d).

Paradoxically, with both congenital and acquired orbital vascular anomalies, haemorrhage occurs far more commonly with low-pressure venous or venouslymphatic ('lymphangioma') anomalies than with highpressure (or high-flow) malformations or fistulae. Bleeds from orbital lymphangiomas often start in childhood (Figure 2e) and appear to become less frequent with age-conjecturally due to perivascular fibrosis in areas of prior haemorrhage. Very rarely, haemorrhage in adulthood can arise from an occult venous anomaly (Figures $2 \mathrm{f}$ and $\mathrm{g}$ ) or from areas of necrosis within an orbital tumour (Figure $2 \mathrm{~h}$ ).

Spontaneous haemorrhages can also occur within the fibrous sheath around solid silicone implants within the orbit, these presenting with acute proptosis and a delay of a week or longer before periocular bruising becomes apparent.

\section{Treatment of spontaneous orbital haemorrhage}

Arterial orbital haemorrhage, whether due to surgery or other trauma, risks a rise in orbital pressure sufficient to stop optic nerve circulation at the orbital apex or in the peripapillary area. ${ }^{6-8}$ In such circumstances, if there is a 

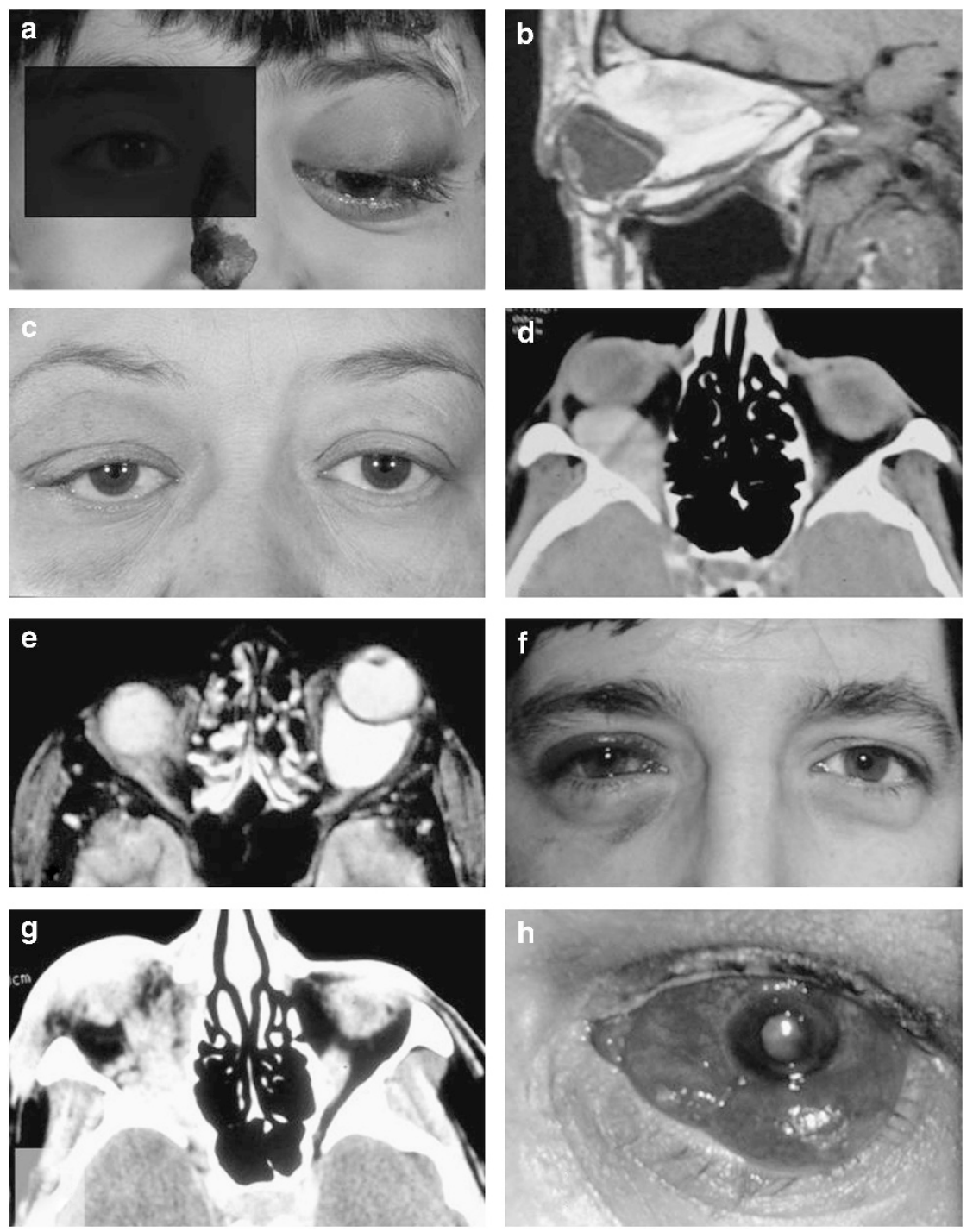

Figure 2 (a) Child with gross left proptosis due to orbital haemorrhage after a blow to his left frontal region during a fall; CT shows a large subperiosteal haemorrhage, arising from a frontal plate fracture, with the mass causing major distortion of the globe (b). (c) Elderly diabetic patient with overnight onset of right periocular swelling and minimal change in ocular motility, this being due to a spontaneous orbital haemorrhage; such vasculopathic haemorrhages typically have a 'beached whale' configuration and occupy the inferior-temporal quadrant (d). (e) Massive loculated left retrobulbar haemorrhage in a 2-year-old child with lymphangioma. (f) Patient with acute onset of right blindness and orbital pain, eyelid bruising appearing 2 days after onset of symptoms; imaging (g) showed a diffuse apical haemorrhage scattered amongst a pre-existing occult venous malformation. (h) Rapid onset of left orbital inflammation secondary to necrosis within an unknown intraocular melanoma.

rapid and dramatic increase in proptosis, marked loss of eye movements and increasing resistance to retropulsion, then treatment should be prompt and thorough. First-in the absence of globe rupture-intermittent very firm pressure should be applied to the orbit to tamponade bleeding from the disrupted artery while it undergoes spasm and secondary treatment is being organised; such pressure should be released at regular intervals to permit optic nerve perfusion. The next phase of treatment should be directed to drainage of the haemorrhage or creation of more space for the orbital contents by disrupting the orbital septum. With acute postoperative haemorrhage, a surgical drain should be gently manipulated to encourage drainage, the incision reopened widely, and the tissues gently spread to encourage release of any fluid or haematoma; prolapse of orbital fat from the incision is of no clinical concern, as this acts to reduce further the orbital pressure.

Two main methods exist for urgently reducing the pressure within an unoperated 'rock-hard' orbit with failing vision. If a likely site of haemorrhage is evident (as with hypoglobus due to haematoma gathering 
alongside a roof fracture; Figure 2a), then a large incision should be placed along the appropriate upper or lower lid skin-crease to allow prolapse of the tissues;
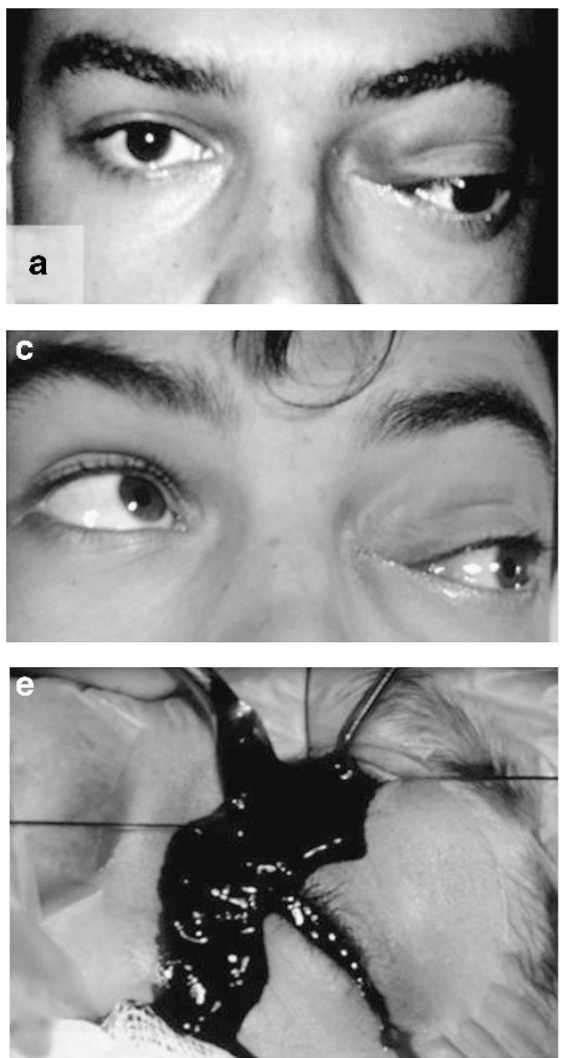

blunt-ended scissors should then be passed backwards into the orbital tissues and spread gently in the upper or lower orbital fat pads to release blood and tissue fluid.
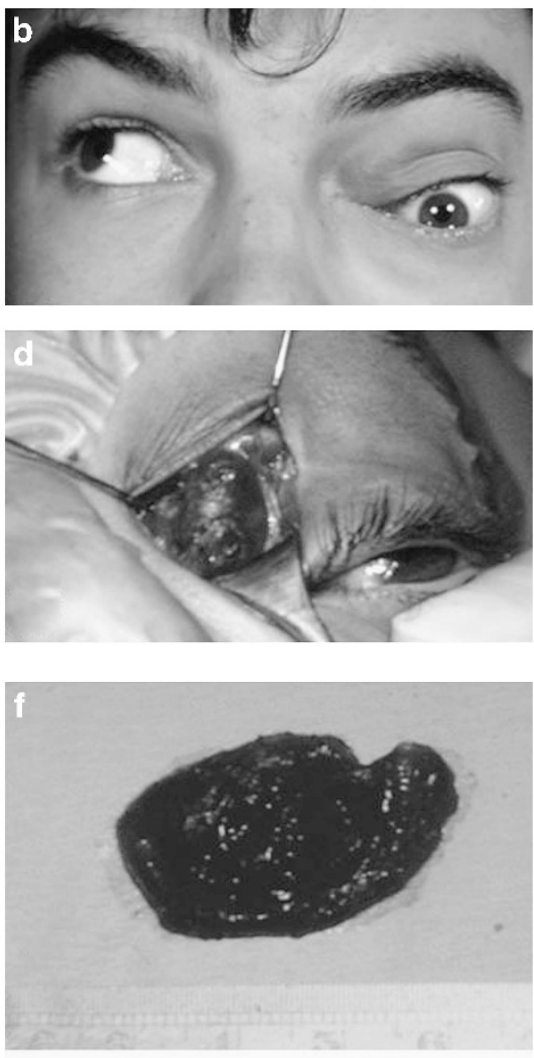

Figure 3 (a) Persistent left hypoglobus, proptosis and lateral displacement, with marked restriction of eye movements (b and c), at 8 months after onset of retrobulbar haemorrhage. At anterior orbitotomy a well-defined encysted orbital haemorrhage was (d) exposed, (e) drained and the fibrotic, blood-stained 'ochre' membrane excised (f).
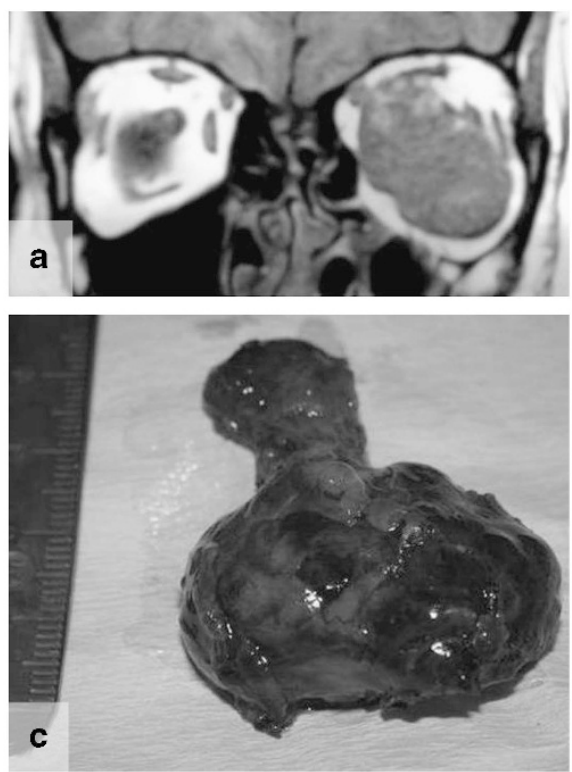
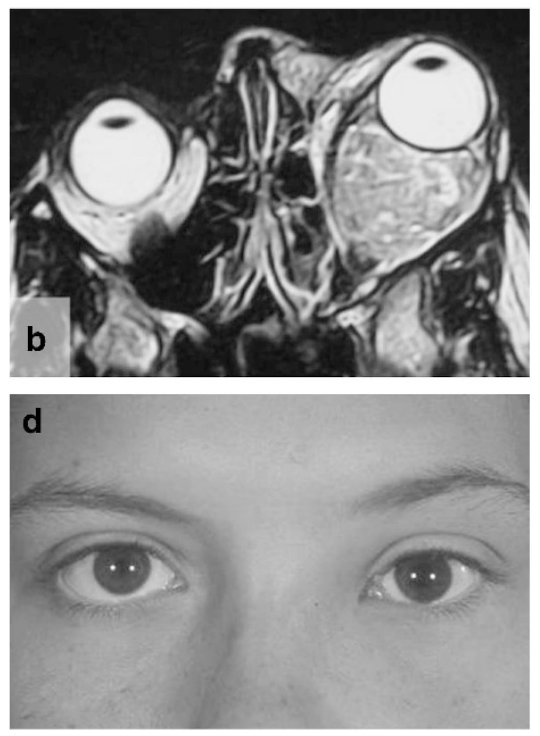

Figure 4 ( $\mathrm{a}$ and $\mathrm{b}$ ) Gross left proptosis due to a longstanding intraconal lymphangioma surrounding the optic nerve. The mass, of 17-ml volume, was excised intact (c) with an acuity of 6/5 and good ocular motility after surgery (d). 
If the focus of haemorrhage is not apparent, a nonspecific release of the orbital septum is provided by wide lateral cantholysis. If such surgery is restricted to a horizontal canthotomy alone, the lateral fixation of the orbital septum will remain attached and nothing will be achieved; the horizontal canthotomy must, therefore, be followed by both upward and downward division of the orbital septum over at least $1 \mathrm{~cm}$ around the rim. A simpler and quicker alternative is to perform an $8-10 \mathrm{~mm}$ full-thickness perpendicular incision through the upper and lower eyelids and deeper opening of the orbital septum at the outer canthus; these incisions generally heal well, and any minor risk of scarring is justified in the bid to save eyesight.

The spontaneous vasculopathic haemorrhage (Figures $2 \mathrm{c}$ and $\mathrm{d}$ ) rarely requires acute intervention and most will reabsorb over many months-although proptosis may increase over the first few weeks due to the increasing osmotic effect of blood breakdown within the encysted haemorrhage. These lesions can be monitored clinically, with repeat imaging considered where there is a persistent deterioration of clinical signs. Stopping anti-platelet agents is probably functionless, as any reduction in action will come too late to affect the original haemorrhage.

Many bleeds within mixed venous-lymphatic malformations will slowly absorb over a few months.

However, where a large encysted haemorrhage is causing persistent optic neuropathy, distorting the globe sufficiently to cause amblyopia, or causing major disfigurement or diplopia (Figures $3 \mathrm{a}-\mathrm{c}$ ), consideration should be given to exploration and drainage of the mass. Although the abnormal blood cyst can be exposed (Figure 3d) and drained (Figure 3e), in many cases further refilling or haemorrhage can be avoided by resection of the ochre membrane that forms around the collection of blood (Figure 3f). Injection occlusive
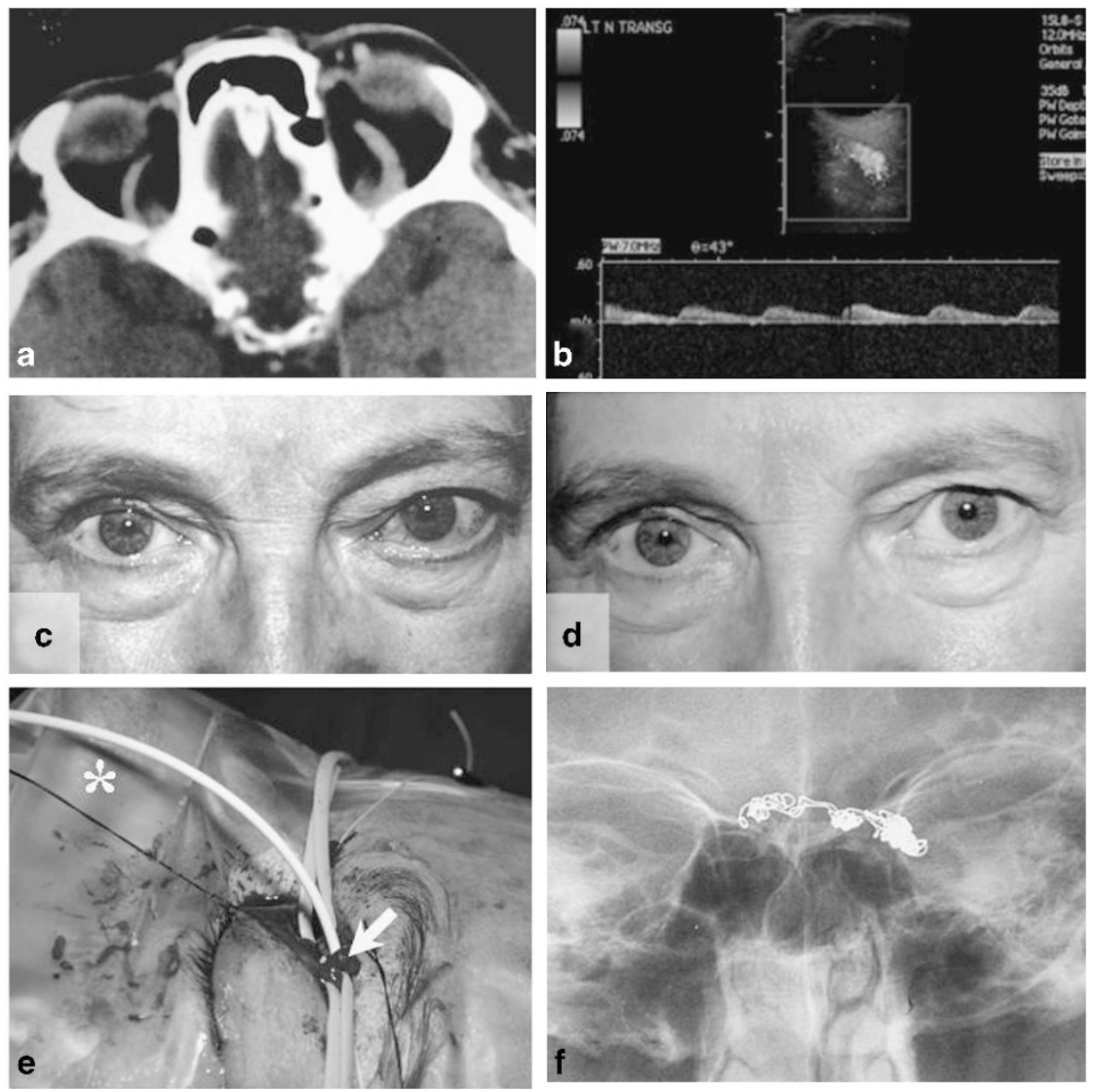

Figure 5 (a) Mild proptosis and enlargement of the left superior ophthalmic vein due to low-flow dural shunt, the enlarged superior ophthalmic vein showing a reversal of flow-direction (from the normal anterior-to-posterior direction) and arterial wave-form on Doppler ultrasonography (b). Spontaneous low-flow dural arterio-venous shunts, typically arising in patients with chronic vascular disease such as diabetes or hypertension, present with acute engorgement of episcleral veins (c); low-flow shunts, in many cases, resolve spontaneously after many months-in this case at 15 months after onset (d), (e) where impairing ophthalmic functions, persistent high-flow shunts into the cavernous sinus can be treated by cannular placement into the deeper extent of the engorged superior ophthalmic vein (asterisk), with retrograde placement of platinum coils (f) to induce thrombosis within the central venous system. 
sclerotherapy provides a useful alternative treatment for persistent lymphatic malformations: large cysts are identified and drained by direct puncture under radiographic control, with instillation of a surface-active agent and alcohol to destroy the endothelial lining and cause gross inflammation, and then a firm occlusive pressure is applied to close the lumen while a vacuum drain is in place. Sclerotherapy will not resolve large mixed venous and lymphatic malformations, and in some cases these massive lesions can be successfully removed intact (Figure 4 ).

\section{Acute vascular shunts in the orbit or periorbital area}

Shunting between the orbital arterial and venous circulations will typically result in acute symptoms and signs, the most obvious being episcleral vascular engorgement (Figure 1b) and chemosis (subconjunctival oedema; Figure 1c). ${ }^{9-13}$ Other signs include a moderately raised intraocular pressure with applanation tonometry identifying an excessive cardiac swing in some patients.

The commonest arterio-venous shunts in the orbital area are so-called 'low-flow' shunts, where an intracranial meningeal arteriole ruptures into the venous circulation in the region of the cavernous sinus, thereby secondarily raising the orbital venous pressure. The diagnosis is confirmed on imaging, where an enlarged superior ophthalmic vein may be associated with mild proptosis and slight enlargement of several extraocular muscles (Figure 5a). Colour Doppler ultrasonography shows a reversal of flow within the enlarged superior ophthalmic vein, this reversal (with flow from posterior to anterior) being during the systolic or, in some cases, the whole of the cardiac cycle (Figure 5b). These low-flow shunts usually occur with systemic vascular disease, and most will close spontaneously after some months
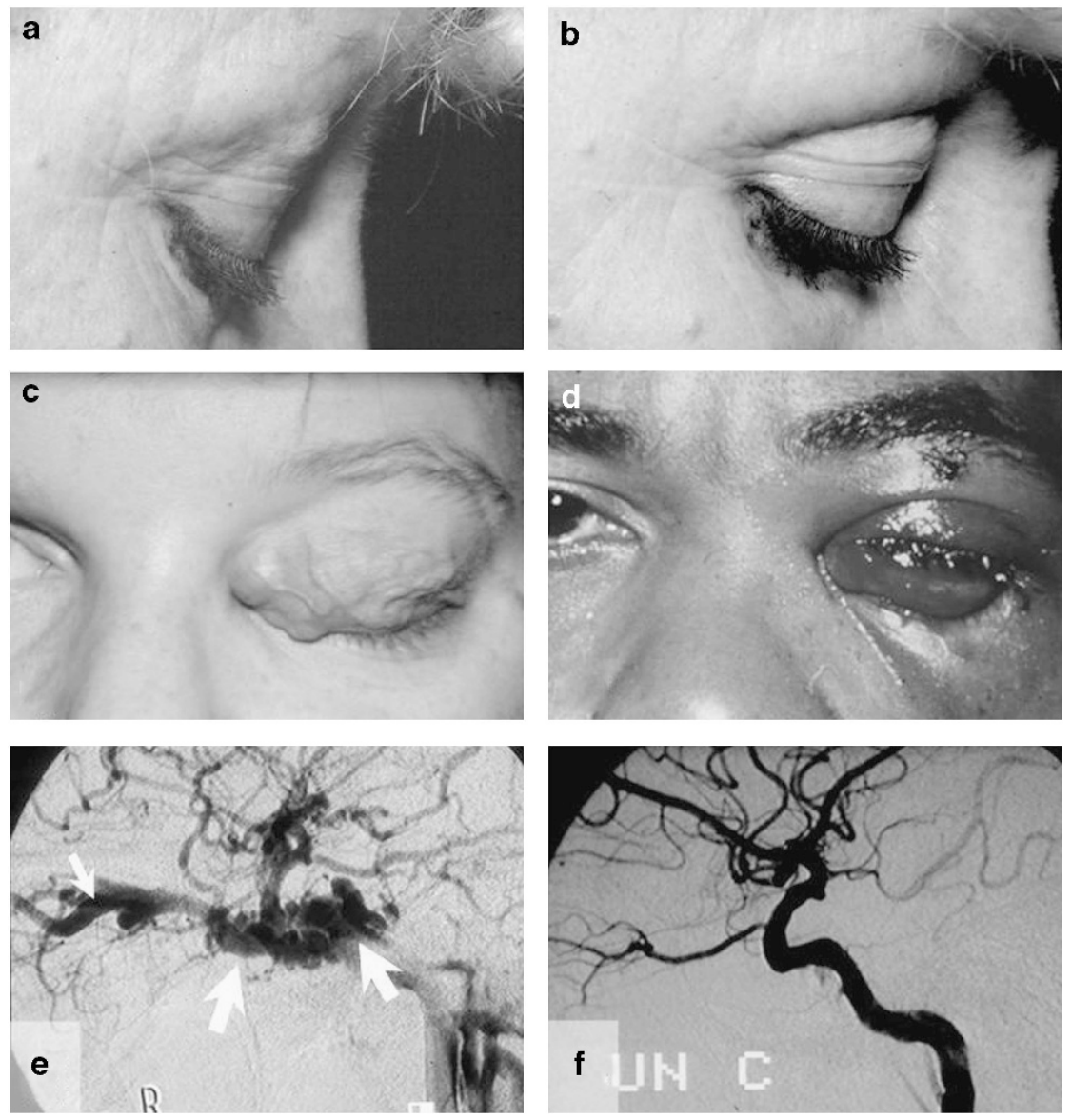

Figure 6 Localised post-traumatic arterio-venous shunt in the right upper lid sulcus, viewed during (a) the systolic phase and (b) diastolic phase of the cardiac cycle. (c) Young adult with left orbital high-flow arterio-venous malformation that has increased in size and effect for 11 years after onset of symptoms. (d) Marked left proptosis, orbital congestion and optic neuropathy due to acute carotico-cavernous fistula after gunshot injury. Carotid angiography (e) demonstrates intense filling of the cavernous sinus (large arrows) during the arterial phase and inappropriate retrograde filling of the superior ophthalmic vein (small arrow); after balloon embolisation (f) there has been closure of the fistula between the carotid siphon and cavernous sinus. 
(Figures $5 c$ and $d$ ); the majority of patients are therefore managed conservatively, with monitoring of visual status, topical antihypertensives if the intraocular pressure is considered too high, and reassurance. However, when flow through the fistula threatens the vision, or there is significant risk of cerebral venous thrombosis, then closure of the shunt should be considered. It is unusual to be able to seal these lesions from the arterial side, and most will be closed by insertion of thrombogenic coils into the cavernous sinus-these coils being inserted through retrograde catheterization of the superior ophthalmic vein (Figures 5e and f).

High-flow arterio-venous shunts are relatively rare within the orbit and often present as a sudden onset of a pulsatile swelling of the eyelid (Figures $6 a$ and b), these shunts are often increasing -in both size and flow-quite dramatically over months to years (Figure 6c). As the orbital arteries comprise a water-shed between the internal and external carotid territories, treatment of such arterio-venous shunts is by selective angiography and endovascular occlusion of the feeding vessels. High-flow carotico-cavernous fistulae-where the intracavernous carotid syphon ruptures into the cavernous sinus-occur after major cranial trauma or in patients with systemic vascular disease. These present with acute onset of major proptosis, conjunctival oedema, restriction of eye movements and visual impairment, and many patients will be aware of a pulsatile rushing sound within their head (Figure 6d). Carotico-cavernous fistulae are closed by endovascular radiographic methods, such as balloon occlusion of the fistula (Figures 6e and f).

\section{Orbital vascular occlusion}

Acute occlusion of orbital vessels, either arterial or venous, is extremely rare and generally arises as a result of underlying vascular anomaly, trauma or periorbital infection.

Patients with known orbital venous malformations can occasionally experience a dramatic worsening of symptoms, and this is either due to haemorrhage from the fragile abnormal vessels or due to thrombosis within a major part of the lesion. Severe secondary orbital congestion can occur (Figures $7 a$ and b), and this can pose a major threat to vision or the eye itself. Although surgery carries a significant risk of damage to normal orbital structures and loss of vision, there is often no realistic alternative to surgical intervention and the results can be gratifying and long-term (Figures 7c and d).

Periorbital infections, particularly very severe sinusitis, can lead to thrombosis of the cavernous sinus or the superior ophthalmic vein, or to an occlusive arteritis with orbital necrosis - the latter particularly with some fungal infections of the sinuses, such as Mucor. Treatment of these life-threatening conditions is undertaken with infectious disease physicians, intensivists, head-and-neck
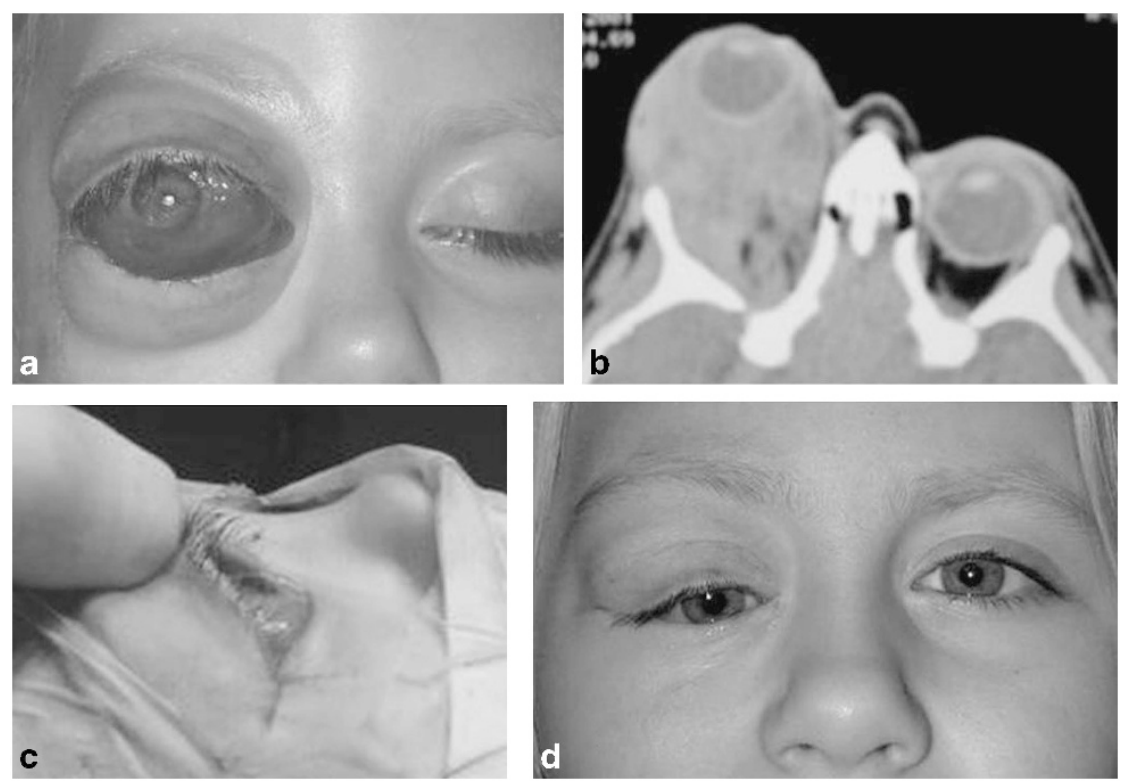

Figure 7 (a) Acute severe proptosis and ocular surface exposure in a 2-year old with known right mixed venous-lymphatic malformation, the child being referred for orbital exenteration. (b) Imaging showed widespread vascular anomalies throughout the orbit and thrombosis within several parts of the lesion-the cause for the acute severe deterioration of signs-facilitated extensive resection of the mass (c). (d) The child at 18 months after resection of the mass, with an amblyopic eye and some residual vascular anomaly in the superotemporal quadrant of the right orbit. 

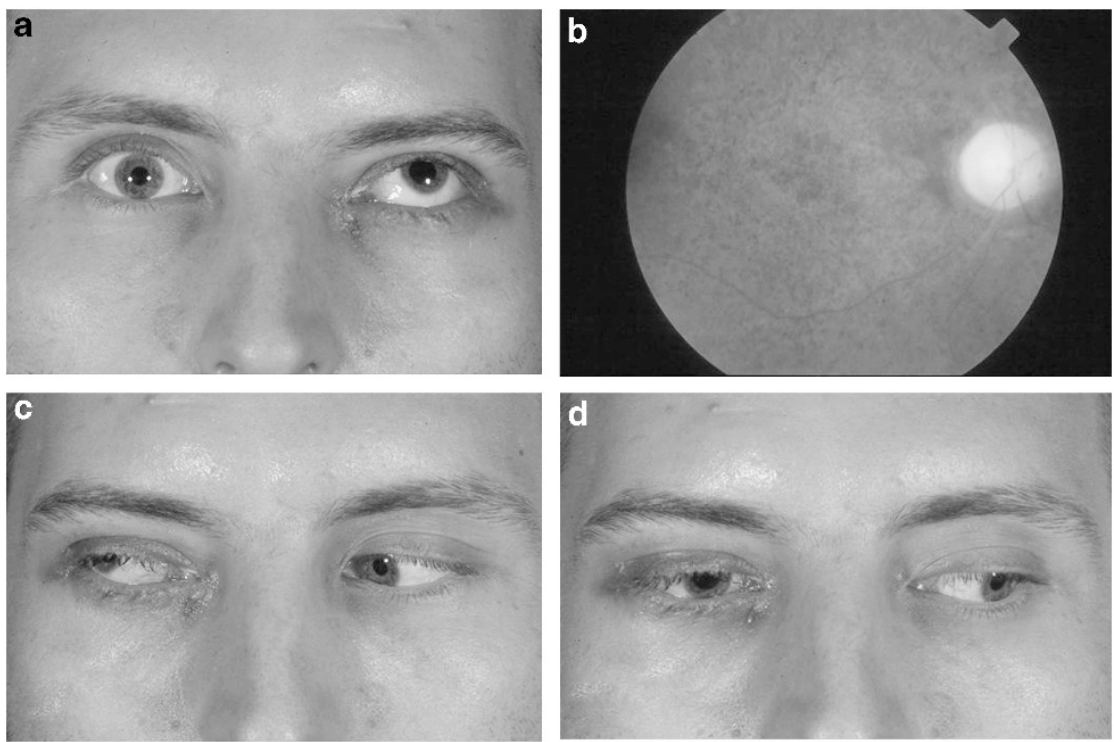

Figure 8 Orbital ischaemic syndrome arising in an intravenous drug user who slept overnight resting continuously on his right orbit. (a) A day after injury, the right eye was blind and he had no movements on the affected side (attempted upgaze). A few months later there was complete optic atrophy (b), but good recovery of eye movements (c and d).

surgeons, and ophthalmologists. Such patients generally require life-supportive measures, surgical drainage, or debridement of infected or necrotic tissues, high-dose systemic antibacterial or antifungal agents, and often anticoagulation.

Isolated occlusion of the orbital arterial circulation may occur as a result of prolonged pressure applied to the orbit, and this can lead to blindness, a global loss of eye movements, a dilated, non-reactive pupil, and an ischaemic anterior uveitis. Blindness after pronepositioning for prolonged neurosurgical procedures has been thought to result from the affected orbit resting on the face-rest for several hours, this having a poor prognosis. The same process can occur in an unconscious individual under the influence of alcohol or other substances after a prolonged period of inadvertent pressure on the globe and orbit (Figure 8).

\section{Summary}

Acute orbital vascular events are most commonly due to haemorrhage, are more rarely due to arterio-venous shunts (the shunt often sited in the periorbital area), and very rarely arise from intraorbital vascular occlusion. The likely diagnosis is often evident on taking a thorough history and examination and in most cases, after appropriate imaging, the process can be monitored for progression. Visual failure is usually due to high pressure at the orbital apex, resulting in ischaemic optic neuropathy, and any evidence of a persistent significant visual impairment should prompt intervention.

\section{Conflict of interest}

The authors declare no conflict of interest.

\section{Acknowledgements}

Our thanks to Mr Alan McNab for use of illustrations in Figures 5e and f. GE Rose receives some funding from the Department of Health's NIHR Biomedical Research Centre for Ophthalmology at Moorfields Eye Hospital and UCL Institute of Ophthalmology.

\section{References}

1 Atalla ML, McNab AA, Sullivan TJ, Sloan B. Nontraumatic subperiosteal orbital hemorrhage. Ophthalmology 2001; 108: 183-189.

2 Sullivan TJ, Wright JE. Non-traumatic orbital haemorrhage. Clin Experiment Ophthalmol 2000; 28: 26-31.

3 Wright JE, Sullivan TJ, Garner A, Wulc AE, Moseley IF. Orbital venous anomalies. Ophthalmology 1997; 104: 905-913.

4 Moin M, Kersten RC, Bernardini F, Kulwin D, Biddinger PW, Ernst RJ et al. Spontaneous hemorrhage in an intraorbital arteriovenous malformation. Ophthalmology 2000; 107: 2215-2219.

5 Gilhotra JS, McNab AA, McKelvie P, O’Donnell BA. Late orbital haemorrhage around alloplastic orbital floor implants: a case series and review. Clin Experiment Ophthalmol 2002; 30: 352-355.

6 Winterton JV, Patel K, Mizen KD. Review of management options for a retrobulbar hemorrhage. J Oral Maxillofac Surg 2007; 65: 296-299.

7 Shek KC, Chung KL, Kam CW, Yau HH. Acute retrobulbar haemorrhage: an ophthalmic emergency. Emerg Med Australas 2006; 18: 299-301. 
8 Burkat CN, Lemke BN. Retrobulbar hemorrhage: inferolateral anterior orbitotomy for emergent management. Arch Ophthalmol 2005; 123: 1260-1262.

9 de Keizer R. Carotid-cavernous and orbital arteriovenous fistulas: ocular features, diagnostic and hemodynamic considerations in relation to visual impairment and morbidity. Orbit 2003; 22: 121-142.

10 Miller NR. Dural carotid-cavernous fistulas: epidemiology, clinical presentation, and management. Neurosurg Clin N Am 2012; 23: 179-192.
11 Fattahi TT, Brandt MT, Jenkins WS, Steinberg B. Traumatic carotid-cavernous fistula: pathophysiology and treatment. J Craniofac Surg 2003; 14: 240-246.

12 Gemmete JJ, Ansari SA, Gandhi DM. Endovascular techniques for treatment of carotid-cavernous fistula. I Neuroophthalmol 2009; 29: 62-71.

13 Tjoumakaris SI, Jabbour PM, Rosenwasser RH. Neuroendovascular management of carotid cavernous fistulae. Neurosurg Clin N Am 2009; 20: $447-452$. 